\title{
Efectividad de una intervención cognitivo-conductual combinada con técnicas de la entrevista motivacional en bulimia nerviosa: un estudio de caso.
} Effectiveness of combined cognitive-behavior
intervention and motivational interviewing techniques
in bulimia nervosa: a case study.

Fecha de recepción: 12-07-2018

Fecha de aceptación: 18-07-2018
Lucía Beltrán Garayo

Facultad de Psicología. Universidad Autónoma de Madrid Ana Rosa Sepúlveda Garcia Facultad de Psicologia. Universidad Autónoma de Madrid.

\section{resumen/alostract:}

El presente trabajo describe el proceso terapéutico a través de una intervención cognitivo-conductual de una mujer de 25 años con un trastorno del comportamiento alimentario, con un diagnóstico de Bulimia Nerviosa. Se utilizó un diseño de caso único y medidas pre-post, con cuestionarios de patología alimentaria como el EDE-0 y BITE, y de bienestar emocional como el BDI-II, el STAl y el DERS. La intervención fue diseñada a partir del análisis funcional del comportamiento con el fin de: mejorar la adherencia al tratamiento, incorporar un patrón de cinco ingestas diarias, reducir el número de conductas compensatorias y episodios de sobreingestas, y mejorar el autoconcepto. Las técnicas empleadas fueron: psicoeducación, técnicas de la entrevista motivacional, auto-registros, modelado, entrenamiento en respuesta alternativa, control estimular, solución de problemas, reestructuración cognitiva, control de la respiración y exposición. La intervención se llevó a cabo durante 20 sesiones, distribuidas semanalmente. A partir de un análisis cualitativo y cuantitativo de la evolución de la paciente, se puede concluir que el tratamiento fue efectivo, y los retos de la intervención son discutidos.

The present work describes the therapeutic process through a cognitive-behavioral intervention of a 25 years old female who presents an eating disorder, diagnosed with Bulimia Nervosa. A single case research design and prepost measures were used, with eating disorder questionnaires such as EDE-Q and BITE, and emotional well-being questionnaires such as BDI-II, STAI and DERS. The goals for treatment, based on the functional analysis, were as follows: improve adherence to treatment, incorporate a pattern of five daily intakes, reduce the number of compensatory behaviors and binges, and improve the patient's self-concept. The techniques used were: psychoeducation, motivational interviewing techniques, self- registers, modeling, alternative response training, stimulus control techniques, problem solving, cognitive restructuring, breathing control techniques and exposure. The treatment was applied in 20 weekly sessions. From the qualitative and quantitative analysis of the information, it can be concluded that the treatment was effective. The challenges of the intervention are discussed.

\section{palabras clave/keywords:}

Estudio de caso único, bulimia nerviosa, intervención cognitivo-conductual, técnicas de la entrevista motivacional. Single case study, bulimia nervosa, cognitive-behavioral intervention, motivational interviewing techniques. 


\section{Introducción}

Los trastornos del comportamiento alimentario (TCA) son enfermedades mentales graves caracterizadas por comportamientos y actitudes patológicas con respecto a la alimentación (Gandarillas, Zorrilla, Sepúlveda, y Muñoz, 2003; Treasure, Claudino y Zucker, 2010), que suelen acompañarse de intensa preocupación por el peso o la figura corporal (APA, 2013). Los TCA son patologías difíciles de tratar que se han asociado con una calidad de vida baja, tasas de comorbilidad alta, y una mortalidad prematura (Herpertz-Dahlmann, 2009). Las personas que padecen estos trastornos comparten una serie de características: la dificultad para aceptarse a sí mismos, la insatisfacción corporal, un miedo extremo a perder el control de su vida, y la dificultad para aceptar su problema (Graell, Villaseñor y Morandé, 2014).

El Manual Diagnóstico y Estadístico de los Trastornos Mentales (DSM-5, APA, 2013) ha establecido en su última edición tres trastornos principales dentro de la categoría TCA: anorexia nerviosa (AN), bulimia nerviosa (BN) y trastorno por atracón (TA). Las alteraciones alimentarias significativas que no cumplen los criterios para estos diagnósticos se han incluido dentro del grupo Trastornos especificados y no especificados de la ingesta y la conducta alimentaria.

Tabla 1. Principales diagnósticos clínicos de TCA, según el manual DSM-5 (APA, 2013).

\begin{tabular}{ccccc}
\hline Síntoma & $\begin{array}{c}\text { AN } \\
\text { (restrictiva) }\end{array}$ & $\begin{array}{c}\text { AN (con atra- } \\
\text { cones/purgas) }\end{array}$ & BN & TA \\
\hline Restricción de la ingesta & Sí & Sí & -- & -- \\
Peso corporal & Bajo $^{2}$ & Bajo $^{2}$ & -- & -- \\
Episodios de atracones & No & Sí & Sí & Si \\
Comportamientos compensa- & No & Sí & Sí & No \\
torios ${ }^{1}$ & & & & -- \\
Miedo intenso a engordar & Sí & Sí & Sí & -- \\
Influencia exagerada del peso/ & Sí & Sí & & \\
figura en la autoevaluación & & &
\end{tabular}

Nota: ${ }^{1}$ Comportamientos compensatorios: vómito autoprovocado, uso de laxantes, diuréticos, ayuno, ejercicio excesivo, etc. ${ }^{2}$ Bajo: significativamente bajo, definido como un peso que es inferior al mínimo normal (APA, 2013)

En los últimos años se ha observado un aumento en la prevalencia de los casos de TCA, especialmente notable en la población de mayor riesgo: mujeres jóvenes y adolescentes de países industrializados (Peláez, Labrador y Raich, 2005). En esta población se han centrado la mayoría de los estudios epidemiológicos en España, los cuales muestran una prevalencia de TCA en torno al 4,5\% en mujeres adolescentes y jóvenes (Insalud, 1995; Peláez, Raich y 
Labrador, 2010). Si atendemos la prevalencia de BN, las cifras mostradas en España coinciden con las de otros países desarrollados, estimándose en un porcentaje de 1-3\% según las principales revisiones epidemiológicas a nivel global (Hoek y van Hoeken, 2003; Smink, Van Hoeken y Hoek, 2012).

Para entender la complejidad del desarrollo de un TCA, es conveniente adoptar una perspectiva multidimensional. Morandé (2014) establece que en estos trastornos existe una transmisión vertical (cultural), horizontal (entre pares) y generacional (vulnerabilidad genética y hábitos familiares). El curso de los TCA es heterogéneo, no obstante, se puede afirmar que existe una tendencia relativa a la cronicidad, esto es, pacientes de larga evolución que muestran resistencia al tratamiento tras años desde el inicio del trastorno (Calvo, 2011; Insalud, 2000). Esta evolución del trastorno a lo largo del tiempo lleva asociado un posible deterioro integral de la persona. No obstante, hablar de cronicidad no supone irreversibilidad (Turón, 2011): a mayor tiempo de seguimiento tras el tratamiento, mayor probabilidad de recuperación total o parcial (Keel y Broen, 2010). Por ello, las intervenciones terapéuticas deben iniciarse inmediatamente tras el diagnóstico, deben tener la frecuencia y duración suficientes como para asegurar la mejora completa de las jóvenes. Requieren la adopción de una perspectiva multidimensional desde la cual se lleve a cabo un plan de acción integral, en el que colaboren distintos profesionales, ofreciendo una serie de servicios específicos para estos trastornos.

La práctica asistencial requiere de una elevada especialización, así como la actualización continuada de los conocimientos científicos. Desde un abordaje psicológico, distintas aproximaciones terapéuticas han mostrado su eficacia en el tratamiento de los TCA. En el caso de la Bulimia Nerviosa, la Terapia Cognitiva-Conductual (TCC) es el tratamiento con mayor apoyo empírico (American Psychiatric Association, 2000). Asimismo, los avances de las últimas décadas muestran que los programas de Autoayuda Guiada (de corte cognitivo-conductual) pueden ofrecer resultados similares a la TCC en ciertos pacientes (Mitchell, et al., 2011). La guía NICE (2017), siguiendo un modelo de cuidados escalonados, recomienda la Autoayuda Guiada como primera opción, seguida por la TCC en caso de no ser efectiva la primera. Faiburn (1985) desarrolló el tratamiento pionero del que parten la mayoría de TCC para la BN, el cual se centra en la modificación de los patrones alimentarios, las conductas de control de peso (purgas, uso de laxantes, diuréticos, dietas estrictas...) y la sobrevaloración excesiva de la figura, el peso y su control. Las pacientes encuentran frecuentemente utilidad en dichos síntomas y expresan algún grado de ambivalencia ante el cambio (Serpell, Treasure, Teasdale y Sullivan, 1999), lo que hace que la motivación al cambio sea una dimensión importante que tratar. El modelo transteórico del cambio, de Prochaska y DiClemente (1984), asume que el cambio de conducta es un proceso gradual, dividido en fases. La entrevista motivacional (EM), articulada por Miller y Rollnick (1991; 2015), es una técnica sofisticada que aporta una herramienta para aumentar la motivación del paciente, ayudando a afrontar situaciones en las que las estrategias habituales que promueven el cambio de conductas no son eficaces sin la motivación necesaria por parte de la paciente. En TCA, Treasure y Schmidt (1997) elaboraron algunas guías clínicas para la intervención de jóvenes con AN y BN combinando las técnicas de la EM y la TCC. 
El objetivo del presente trabajo es describir una secuencia de tratamiento que combina EM y TCC en un caso de BN, así como los resultados de dicho tratamiento que apoyan su eficacia.

\section{Descripción del caso}

\section{Identificación de la paciente}

L.P. es una mujer de nacionalidad española, tiene 25 años, está soltera, y vive con sus padres. Ha obtenido una Licenciatura y actualmente cursa un Máster, el cual compagina con trabajos temporales. Es la pequeña de dos hermanas, aunque la hermana mayor, con la cual mantiene una buena relación, no reside actualmente en el domicilio familiar. L. P. mantiene una relación distante con sus padres, refiriendo haber sufrido maltrato psicológico por parte de su padre desde pequeña, al igual que el resto de las mujeres de su familia. Desde hace cinco años, la madre fue diagnosticada de una enfermedad mental grave, encontrándose actualmente en tratamiento.

\section{Motivo de consulta}

La demanda principal según la paciente es la falta de control con la comida, no siendo capaz de seguir la dieta que a ella le gustaría, por lo que presenta episodios de atracones tras los cuales se produce la conducta de vómito auto-inducido. Refiere que en los últimos meses ha aumentado la frecuencia de atracones y purgas, llegando a una frecuencia diaria, lo cual ha aumentado el grado de interferencia en su vida. Acude junto a su hermana, con la cual mantiene una buena relación y a la cual ha explicado su problema, a diferencia de a sus padres. En el momento de realizar la primera sesión está tomando paroxetina, recetado por el médico de cabecera, ante la descripción de la paciente de síntomas depresivos y ansiosos. En el momento de la evaluación, la paciente pesa $54 \mathrm{~kg}$ y mide $1.57 \mathrm{~m}$, lo que resulta en un Índice de Masa Corporal (IMC) de 22.

\section{Evaluación del caso}

Se extendió durante las primeras 2 sesiones, con una periodicidad semanal. Para ello se hizo uso de diversas herramientas:

Entrevista semiestructurada. Las áreas evaluadas son: motivo de consulta, peso corporal, conducta alimentaria, imagen corporal, tratamientos previos, historial médico, historia familiar, historia social y del desarrollo, y síntomas comórbidos.

Así mismo, se emplearon una serie de cuestionarios, que podrían dividirse en dos grupos. Aquellos específicos para la evaluación de los TCA:

EDE-Q (Cuestionario Examen de Trastornos de la Conducta Alimentaria). Desarrollado por Fairburn y Beglin (1994). Es un instrumento de auto-informe de 38 ítems que evalúa, mediante cuatro subescalas, restricción dietética, preocupación por la comida, preocupación por el peso y preocupación por la figura corporal en los últimos 28 días; además de un puntuación global. La versión en castellano validada en población española (Peláez-Fernández, Labrador y Raich, 2013) presenta una consistencia interna satisfactoria en las cuatro subescalas y en la puntuación global del EDE-Q $(\alpha \geq 0,81)$. 
BITE (Test de Investigación de la Bulimia de Edimburgo). Diseñado por Henderson y Freeman (1987). Cuestionario autoadministrado que evalúa la presencia de sintomatología bulímica. Consta de 36 ítems que forman dos subescalas: escala de síntomas y gravedad. La fiabilidad entre items es de 0,96 para la escala de síntomas y de 0,62 para la de gravedad. Versión adaptada y validada en español por Rivas, Bersabé y Jiménez (2004).

Aquellos que abarcan otras áreas de interés para la evaluación psicopatológica:

BDI-II (Inventario de Depresión de Beck-II). Creado por Beck, Steer y Brown (1996). Autoinforme compuesto por 21 ítems de tipo Likert, cuyo objetivo es la detección y evaluación de la gravedad de la depresión. La adaptación al español (Sanz, Navarro y Vázquez, 2003) muestra una buena consistencia interna $(\alpha=0,89)$.

DERS (Escala de Dificultades de la Regulación Emocional). Desarrollada por Gratz y Roemer (2004), demostró una alta consistencia interna $(\alpha=0,93)$ y buena fiabilidad test-retest $(\mathrm{Q}=0,88, \mathrm{p}<, 01)$. Adaptación española por Hervás y Jódar $(2008)$. Evalúa cinco factores: desatención, confusión, rechazo, interferencial y descontrol, mediante 28 ítems con una escala tipo Líkert de cinco puntos.

STAI (Inventario de Ansiedad Estado-Rasgo). Desarrollada por Spielberger, Gorsuch y Lushene (1970). Cuestionario autoadministrado que consta de 40 ítems con una escala tipo Líkerts de 4 puntos, que evalúa ansiedad como estado y ansiedad como rasgo. Se ha empleado la adaptación española de Buela-Casal, Guillén-Riquelme y Seisdedos Cubero (2011); la cual presenta una consistencia interna $\alpha$ de entre 0,89-0,95 en ansiedad estado $(\mathrm{A} / \mathrm{E})$, y entre 0,82-0,91 en ansiedad rasgo (A/R).

\section{Formulación clínica}

Historia del problema/Hipótesis de adquisición. L.P. refiere haber estado preocupada por el peso "desde siempre". Desde pequeña realiza dietas restrictivas junto a su hermana, y hacia los 14 años comenzó a hacer uso de diuréticos e ir al gimnasio con el fin de adelgazar. Los logros referidos a la pérdida de peso recibieron la aprobación familiar, lo que produce una asociación por reforzamiento positivo entre dieta, ejercicio excesivo y uso de diuréticos con bienestar y una mayor autoestima. Las dietas restrictivas aumentan a los 18 años, momento en el que la paciente sitúa el inicio del trastorno, coincidiendo con la ruptura de una relación sentimental con una persona que la insulta por su físico, atribuyendo la paciente su imagen corporal como causa del abandono. A partir de este momento, además del reforzamiento positivo asociado a la dieta, cobra relevancia el mecanismo contrario, es decir, el castigo ante la conducta de comer alimentos calóricos.

Se hipotetiza que esta asociación aversiva lleva a que L.P. mantenga conductas de evitación ante estos alimentos, convirtiéndose en "alimentos prohibidos". Esta asociación a su vez lleva a que la paciente experimente sentimientos de culpabilidad cada vez que los ingiere, elicitando verbalizaciones de fracaso personal. Por último, la evitación constante de alimentos calóricos a los que se somete la paciente aumenta el valor reforzante de estos alimentos.

Hipótesis de mantenimiento. Tras los periodos de restricción, se producen una serie de "fracasos" al comer alimentos prohibidos, discriminada tal conducta por emociones que la 
paciente experimenta como negativas, tales como la soledad o el sentimiento de rechazo; la conducta de ingesta se ve reforzada negativamente mediante la disminución de las emociones negativas previamente nombradas. Sin embargo, tras este reforzamiento inmediato, aumenta la emocionalidad negativa y surgen las alteraciones cognitivas como "estoy como una foca", "si como pan nunca voy a estar delgada", etc. La culpa y el malestar experimentado finalmente facilitan verbalizaciones de permiso: "qué más da un bollo más" junto a la anticipación del vómito, lo que discrimina la conducta de atracón, que se mantiene por el valor reforzante de la comida. El atracón lleva a una serie de sensaciones, condicionadas (culpa) e incondicionadas (malestar intestinal) que discriminan el vómito, el cual es reforzado negativamente por la disminución de tales sensaciones. Como respuesta a estos episodios de sobreingestas, que a su vez exacerban la preocupación por la figura y el peso, L.P. comienza un nuevo periodo de restricción alimentaria, reforzado nuevamente por la sensación de autocontrol, generándose así un círculo en el cual la dieta propicia el episodio de sobreingesta, y es a su vez la respuesta a éste.

A continuación, se presenta la formulación clínica del caso (Figura 1)

Figura 1. Formulación clínica del caso.

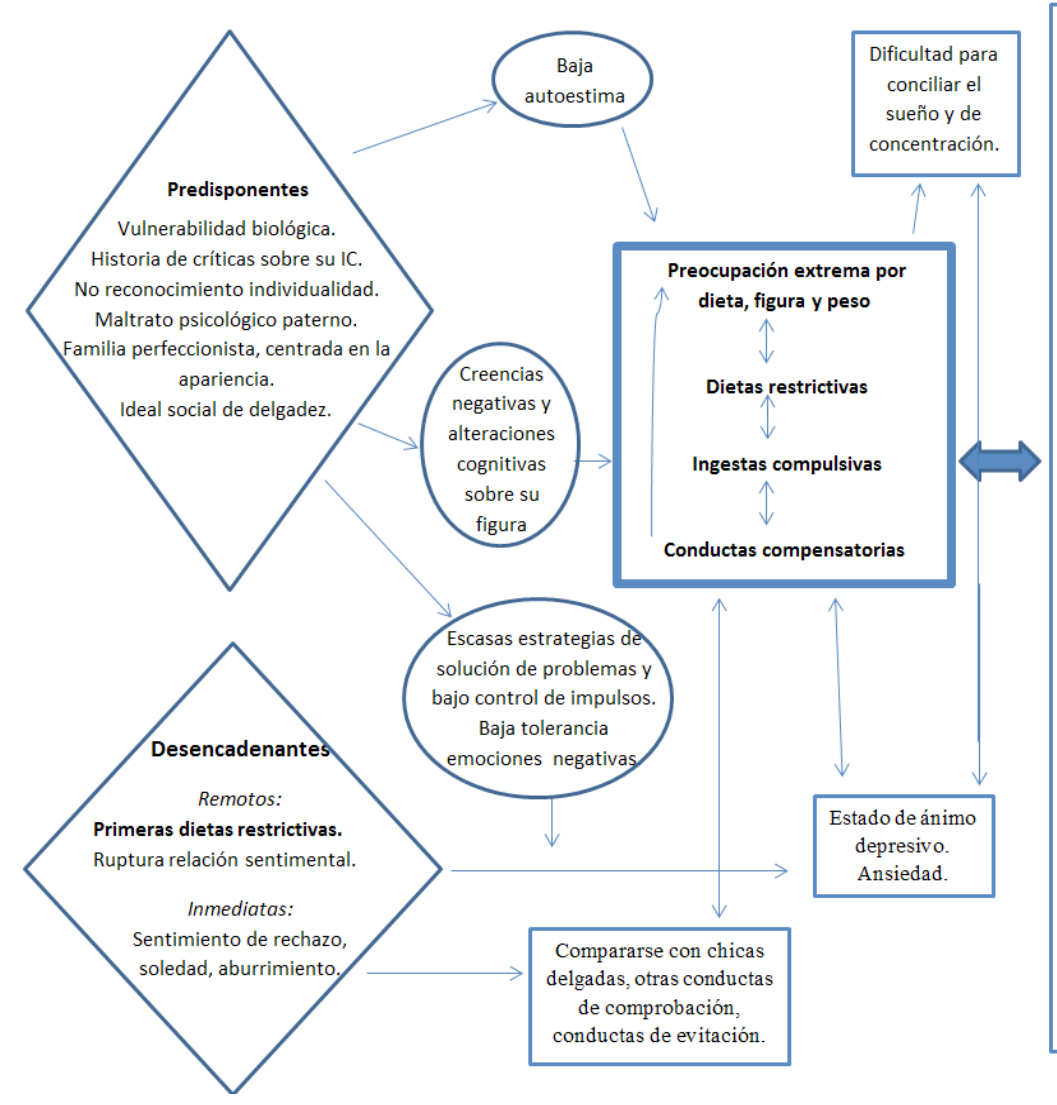

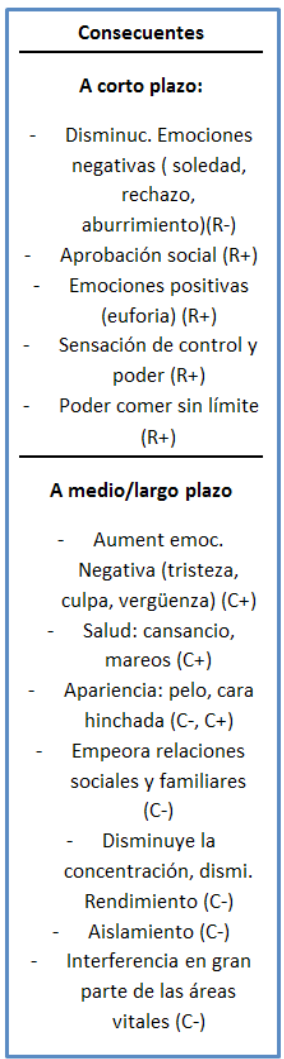




\section{Diagnóstico}

Según la información que se pudo recoger y contrastar mediante las entrevistas, cuestionarios y auto-registros, presenta un diagnóstico clínico de (F50.2) Bulimia Nerviosa [307.25], según los criterios DSM-5 (APA, 2013).

\section{Diseño del estudio}

Se trata de un experimento de caso único $(\mathrm{N}=1)$, en el cual se utiliza un diseño sin retirada A-B (Montero y León, 2007). Por lo tanto, el individuo es el propio control, y se mide el cambio/efecto que produce el tratamiento sobre los objetivos terapéuticos establecidos.

\section{Intervención}

\section{Objetivos del tratamiento}

Se plantea como objetivo central de la terapia la supresión de la sintomatología alimentaria característica del trastorno por Bulimia Nerviosa.

Objetivos específicos. 1. Incrementar y mantener la adherencia al tratamiento (en base a la búsqueda de la motivación al cambio de la paciente). 2. Incorporar un patrón alimentario de cinco ingestas diarias. 3. Reducir el número de atracones. 4. Reducir el número de conductas compensatorias dirigidas a la pérdida de peso (vómitos autoinducidos, uso de diuréticos y dietas restrictivas). 5. Mejorar su autoconcepto, no centrándose únicamente en la figura y el peso.

\section{Aplicación del tratamiento}

El tratamiento se llevó a cabo en 20 sesiones semanales de 60 minutos. En todas ellas se toma el peso de la paciente, sin informarla directamente del mismo; se toma el IMC como indicador de salud (Fairburn, 2008). Además, quincenalmente se tienen sesiones conjuntas con la hermana de la paciente, las cuales posibilitan ampliar la información, y supone un apoyo directo a la paciente. A continuación, se describe el contenido trabajado en las sesiones, según el tipo de técnica empleada:

\section{Técnicas de Psicoeducación}

Empleo de métodos inadecuados para controlar el peso: dieta como desencadenante y mantenedor de los episodios de atracones, la conducta de vómito como conducta compensatoria, mitos e ineficacia del vómito autoinducido y de los diuréticos para controlar el peso.

Consecuencias de la $B N$ : problemas físicos comúnmente asociados a la BN (atracones, vómito autoinducido y uso de diuréticos), como son pérdidas dentales, de pelo, úlcera de hiato, problemas electrolíticas/cardiaco, problemas renales por la deshidratación.

El peso: IMC como indicador de salud, teoría del set-point.

Curva de la ansiedad y su sistema tripartito de respuesta.

Autoevaluación, autoconcepto, autoestima: diferencias y relación entre conceptos, aspectos importantes sobre los que las personas podemos juzgarnos y cómo en los TCA existe una idea sobrevalorada acerca de la figura y el peso. 
Conductas de comprobación y evitación: mantenedoras del problema.

Dieta estricta: tipos, círculo dieta-ingesta compulsiva, efectos de hacer dieta.

\section{Técnicas desde la entrevista motivacional}

Dirigidas principalmente a las conductas de restricción alimentaria, vivenciadas por la paciente como egosintónicas, con el fin de externalizar la enfermedad de la propia persona. Las tareas incluidas son las siguientes:.

1. Creación de una lista de pros/contras actuales del cambio.

2. Creación de una lista de pros y contras futuros del cambio, ¿qué te gustaría que pasará dentro de 5 años?, ¿qué te gustaría que se dijera de ti?

3. El coste de cambiar, ¿qué pierdo o no sé comunicarme ahora de otra manera?

4. Discusión sobre la autoeficacia percibida, las posibles barreras, y la toma de acción.

\section{Técnicas conductuales}

Autoobservación y auto-registros. El objetivo es, por una parte, que la paciente adquiera información acerca de su problema alimentario, y por otra, que esta adquisición de conciencia del problema ayude al cambio. Para ello se hace uso de un registro de control alimentario, extraído de Fairburn (1995), en el que constan los siguientes apartados por ingesta: hora, comida y bebidas consumida, lugar, si la paciente considera esa ingesta excesiva, el empleo de vómito autoinducido o laxantes, y la última columna está reservada para cualquier incidencia que influya en las comidas, así como los sentimientos que se producen antes y después de la ingesta.

Instauración patrón alimentario de 5 comidas: Moldeamiento. El objetivo será hacer cinco ingestas planificadas al día. Dado que L.P. presenta un patrón completamente desestructurado de ingestas, la instauración de las cinco comidas diarias supondrá regular el circuito de hambre-saciedad, mientras a su vez, supondrá la desaparición de uno de los métodos de restricción alimentaria. La instauración de las ingestas se hizo de forma gradual, comenzando por el desayuno y la comida, las cuales le suponían menor dificultad; a continuación, se introdujo la cena; y por último los tentempiés (almuerzo y merienda).

Entrenamiento en respuesta alternativa. Esta técnica de autocontrol se emplea principalmente para el manejo de los impulsos de comer compulsivamente. Consiste en entrenar a la paciente para que ponga en práctica conductas que interfieren o impiden la conducta problema (episodios de sobreingestas). En primer lugar, se le pide que confeccione una lista con todas las actividades alternativas, incompatibles con comer, que se le ocurran. Se promueven aquellas que implican acción. Una vez elegidas, se le pide que las escriba en un papel tamaño tarjeta, para que lo pueda tener siempre a mano, y las ponga en práctica ante el impulso de comer compulsivamente.

Técnicas de control estimular. Cambios en el medio social: se utiliza la presencia de otras personas como medio de influir en el comportamiento. Restringir los estímulos discriminativos: evitar el contacto innecesario con la comida, no llevar más dinero del necesario, limitar la comida disponible...Presentar estímulos discriminativos que incrementen la proba- 
bilidad de aparición de la conducta que se quiere instaurar: llevar lista de la compra, llevar el tentempié preparado al trabajo...Modificar las condiciones fisiológicas: hacer la compra después de comer, no dejar que pasen más de 3-4 horas sin comer, entre otros. Cambios en la secuencia habitual de conductas: se indica a la paciente que ponga los cubiertos en el plato una vez introducido el alimento en la boca, que mastique suficientemente la comida antes de tragar, y que vuelva a repetir este patrón; se consigue de este modo cortar una ingesta excesivamente rápida, que podría provocar una atracón al tener la paciente una sensación de saciedad reducida al acabar la comida e ingerir cantidades excesivas de alimento.

Técnica de solución de problemas. Se plantea esta técnica como modo de afrontamiento de situaciones problemáticas y se analizó, mediante los autorregistros, cómo las ingestas compulsivas tienden a darse en momentos de estrés. Se muestra a la paciente el procedimiento y se practica con ella. Se le pide que ponga en práctica la técnica en su vida diaria, prestando especial atención a aquellas situaciones que suelen generar problemas alimentarios; para ello, cuando surja un problema debe escribirlo en la columna 6 (contexto y comentarios) del registro diario de alimentación, y describir en el reverso de la página los pasos que ha seguido para resolverlo. Por último, debe hacer una revisión del proceso seguido al día siguiente.

\section{Técnicas de reestructuración cognitiva}

Se proporciona información sobre la relación entre cogniciones, conducta y emociones. Se hace especial énfasis en cómo actúan los pensamientos como mediadores del malestar emocional. Se pregunta por estas dificultades de pensamiento/creencias y se verbalizan en la sesión 13 "me estoy poniendo obesa" por las dificultades de seguir las cinco ingestas. Se decide trabajar sobre este pensamiento:

Cuestionamiento socrático: ¿En qué momento se comienza a estar obeso? ¿puede la obesidad concretarse en un peso específico? ¿puede pasarse a estar obeso de un día para otro?, entre otras.

Generación de contraargumentos: ¿considerarías que otras personas con tu talla o peso están obesas? Aquí se analiza si se aplican las mismas normas rígidas y estrictas a sí misma.

Identificar los errores de atribución: hemos visto como el peso fluctúa a lo largo del día y del mes sin que ello signifique un cambio de peso global, ¿puede deberse esa sensación de estar aumentando de peso a otros factores que no sean la realización de las cinco ingestas diarias?

Prueba de realidad: se trabaja con la paciente en terapia este pensamiento, mostrándole la gráfica del IMC.

Generación de pensamiento alternativo. Ej. "que me sienta más gorda ahora mismo no quiere decir que lo esté".

Además, se analizan las formas de pensamiento irracional subyacentes a los pensamientos negativos automáticos. Una vez identificados, se procede al debate socrático de sus ideas irracionales, y se trabaja la generación de pensamientos alternativos. Se emplea la metáfora del reproductor de DVD (extraída de Fairburn, 2008) para explicar que aprender a detectar cuándo se está reproduciendo el DVD de los problemas alimentarios para parar a tiempo y 
seguir lo aprendido durante la terapia. Se trabaja la identificación de los estímulos que activan dichos pensamientos.

\section{Técnica de control de la respiración}

Se introduce esta técnica como método para facilitar la exposición, mediante la disminución de la hiper-activación fisiológica que ésta pueda provocar en los primeros momentos. La elección de esta técnica de relajación frente a otras se debe a su mayor rapidez de aprendizaje y facilidad de utilización en la vida cotidiana.

\section{Técnica de exposición}

La técnica de exposición seleccionada es la auto-exposición, con el fin de introducir aquellos alimentos "prohibidos" para la paciente en su vida diaria. Los alimentos prohibidos son tratados como estímulos fóbicos, cuya evitación mantiene el miedo a ingerirlos. La clave será impedir que la evitación se convierta en una señal de seguridad. A continuación, se describen los pasos a seguir:

1- Confección lista de alimentos prohibidos: ir a un supermercado y apuntar las comidas que le gustan, pero es reacia a comer por el efecto que tenga en su figura o peso.

2- Jerarquizarlos en función del malestar que le produzcan.

3- Introducirlos en las comidas, al principio en aquellas en las que la paciente se sienta cómoda. Se prepara la situación donde se van a ingerir estos alimentos antes de hacerlo.

4- $\mathrm{Al}$ principio la cantidad de comida no es importante, aunque la meta es que la paciente coma cantidades normales.

Se continúa hasta que no presenta ansiedad sobre la comida. Una vez logrado, puede elegir si desea ingerirlos o, por lo menos, no llevar a cabo una dieta tan estricta.

\section{Prevención de recaídas}

En esta etapa se comprueba que las expectativas de L.P. son realistas, se le enseña a distinguir una "caída" de una recaída, y se repasan los logros alcanzados y las estrategias que han sido más útiles a lo largo de la terapia. A su vez, se repasan los factores de riesgo y se prepara un plan de acción en caso de que surjan. Por último, se diseña un plan de mantenimiento de estas posibles recaídas a largo plazo.

A continuación, se presenta para cada sesión la relación entre el trabajo realizado, las tareas para casa y los objetivos terapéuticos trabajados (Tabla 2).

\section{Resultados}

\section{Análisis cualitativo}

El análisis del discurso verbal de la paciente es un indicador útil para valorar el cambio de actitudes y comportamientos producidos a lo largo de la terapia.

Con relación a la mejora de la adherencia al tratamiento (incremento de la motivación al cambio) - Objetivo 1-, hay un cambio en las verbalizaciones respecto a la eliminación de 
Tabla 2. Relación entre los objetivos, las técnicas y trabajo realizado.

\begin{tabular}{|c|c|c|c|}
\hline $\begin{array}{l}\text { S e s - } \\
\text { ión }\end{array}$ & Objetivos & Técnicas incorporadas por sesión & $\begin{array}{l}\text { Tareas entre } \\
\text { Sesiones }\end{array}$ \\
\hline 3 & $1,3,4$ & $\begin{array}{l}\text { Explicación del AF. } \\
\text { Psicoeducación: métodos inadecuados para control } \\
\text { del peso y su papel mantenedor del TCA. }\end{array}$ & $\begin{array}{l}\text { Autorregistro ali- } \\
\text { mentario. }\end{array}$ \\
\hline 4 & $\begin{array}{l}1,2 \\
3,4\end{array}$ & $\begin{array}{l}\text { Introducción patrón regular de comidas. } \\
\text { Psicoeducación: consecuencias de la BN. }\end{array}$ & $\begin{array}{l}\text { Autorregistro ali- } \\
\text { mentario } \\
\text { Carta amigo/enemi- } \\
\text { go BN. }\end{array}$ \\
\hline 5 & 1,2 & $\begin{array}{l}\text { Técnicas de control estimular. } \\
\text { Balance decisional del cambio en el presente. }\end{array}$ & $\begin{array}{l}\text { Autorregistro ali- } \\
\text { mentario }\end{array}$ \\
\hline 6 & 1,3 & $\begin{array}{l}\text { AF purgas. } \\
\text { Balance decisional del cambio en el futuro. }\end{array}$ & $\begin{array}{l}\text { Autorregistro ali- } \\
\text { mentario }\end{array}$ \\
\hline 7 & 3,4 & $\begin{array}{l}\text { AF episodios de sobreingesta. } \\
\text { Psicoeducación: curva de la ansiedad e IMC. } \\
\text { Planificación actividades alternativas. }\end{array}$ & $\begin{array}{l}\text { Autorregistro ali- } \\
\text { mentario }\end{array}$ \\
\hline 8 & 3 & $\begin{array}{l}\text { AF dieta restrictiva. } \\
\text { Psicoeducación: el peso natural. }\end{array}$ & $\begin{array}{l}\text { Autorregistro ali- } \\
\text { mentario }\end{array}$ \\
\hline 9 & 3,4 & $\begin{array}{l}\text { Técnica de resolución de problemas. } \\
\text { Revisión del progreso: avances y barreras. }\end{array}$ & $\begin{array}{l}\text { Autorregistro ali- } \\
\text { mentario. Resolución } \\
\text { de Problemas. }\end{array}$ \\
\hline 10 & $3,4,5$ & $\begin{array}{l}\text { Psicoeducación autoevaluación, autoconcepto, au- } \\
\text { toestima. "Tarta de la autoestima" }\end{array}$ & $\begin{array}{l}\text { AA, RP. Lista } \\
\text { actividades gratifi- } \\
\text { cantes. }\end{array}$ \\
\hline 11 & $3,4,5$ & $\begin{array}{l}\text { Planificación de actividades gratificantes. } \\
\text { Psicoeducación conductas de comprobación y evit- } \\
\text { ación. }\end{array}$ & $\begin{array}{l}\text { AA, RP. Autorregis- } \\
\text { tro de conductas de } \\
\text { comprobación. }\end{array}$ \\
\hline 12 & $3,4,5$ & Abordaje cognitivo conductas de comprobación. & AA, RP. \\
\hline 13 & 5 & $\begin{array}{l}\text { Abordaje cognitivo "sentimiento de gordura". } \\
\text { Identificación creencias mantenedoras del TCA. } \\
\text { Metáfora reproductora de DVD. }\end{array}$ & $\begin{array}{l}\text { Autorregistro ali- } \\
\text { mentario } \\
\text { Resolución Prob- } \\
\text { lemas. }\end{array}$ \\
\hline 14 & 3,5 & $\begin{array}{l}\text { Psicoeducación: dieta estricta, triple sistema de } \\
\text { respuesta de la ansiedad y técnica de exposición. } \\
\text { Abordaje cognitivo sobrevaloración control alimen- } \\
\text { tario. } \\
\text { Entrenamiento respiración diafragmática. }\end{array}$ & $\begin{array}{l}\text { Lista alimentos } \\
\text { prohibidos. } \\
\text { Práctica respiración } \\
\text { diafragmática. }\end{array}$ \\
\hline
\end{tabular}


15-18 3, Jerarquía alimentos prohibidos (4 grupos). Exposición a alimentos prohibidos. Reestructuración cognitiva.

Nota: AA: autorregistro alimentario; AF: análisis funcional; RP: resolución de problemas

las conductas de dieta restrictiva. Mientras en las primeras sesiones verbalizaba "yo lo que quiero es comer poco sin atracones ni vómitos", a partir de la sexta sesión verbaliza "si salir de la BN implica no saltarme comidas entonces lo haré". Si bien han existido fluctuaciones en este aspecto durante la terapia, se ha vuelto a trabajar en este objetivo cada vez que han surgido obstáculos. El resultado ha sido la posibilidad de trabajar y observar los cambios en el resto de los objetivos.

En cuanto al Objetivo 2, incorporar un patrón alimentario de cinco ingestas diarias, se observa un cambio radical en el discurso de la paciente. Mientras que al comienzo indicaba "soy un desastre, me salto la comida y al final termino comiendo un montón de chucherías de la máquina expendedora", al finalizar la terapia el discurso es completamente distinto "voy a comer a casa temprano, y me llevo preparada la merienda a clase".

Respecto al Objetivo 3, reducir el número de conductas compensatorias dirigidas a pérdida de peso, L.P. refiere abandonar el uso de diuréticos de forma inmediata tras la psicoeducación sobre la infectividad de este método, además hace referencia a las consecuencias en su salud que el uso prolongado de éstos podría tener. De igual modo, expresa el temor a las consecuencias físicas de los vómitos. Por último, en cuanto a las dietas restrictivas, L.P. refiere ser capaz de tomar alimentos prohibidos para ella durante años, no evitando cuando abandona el tratamiento casi ningún tipo de comida, aunque sigue restringiendo la cantidad ingerida de dichos alimentos.

En cuanto a la reducción del número de atracones (Objetivo 4), la paciente expresa una reducción progresiva en la cantidad y frecuencia de estos episodios, verbalizando en la última sesión una frecuencia de uno a la semana y en cantidades que podrían ser consideradas normales (ej: seis galletas), aunque la paciente sigue experimentando pérdida de control en estas ingestas no panificadas.

Por último, con relación a su autoconcepto (Objetivo 5: mejora del autoconcepto), la paciente expresó gran asombro ante la sobrevaloración que le atribuía a la figura y el peso ("siempre había sabido que le daba mucha importancia pero al verlo reflejado en el dibujo me ha impactado, incluso me agobia mirarlo"), y tomó un papel activo en la ampliación de las áreas que influían en este aspecto, aumentando el número de actividades agradables y siendo capaz de atribuir valía a estas otras áreas de desarrollo personal.

Además, en paralelo, se observan cambios en su estado de ánimo, tanto en su expresión no verbal como en su discurso. Al finalizar la terapia expresa que ha sido capaz de disfrutar 
de momentos sin pensar por un rato en qué dirán de su cuerpo. También se observa una disminución de la ansiedad, especialmente visible ante acontecimientos relacionados con la comida.

Figura 2. Puntuaciones PRE-POST en la sintomatología alimentaria, obtenidas a través del cuestionario EDE-Q.

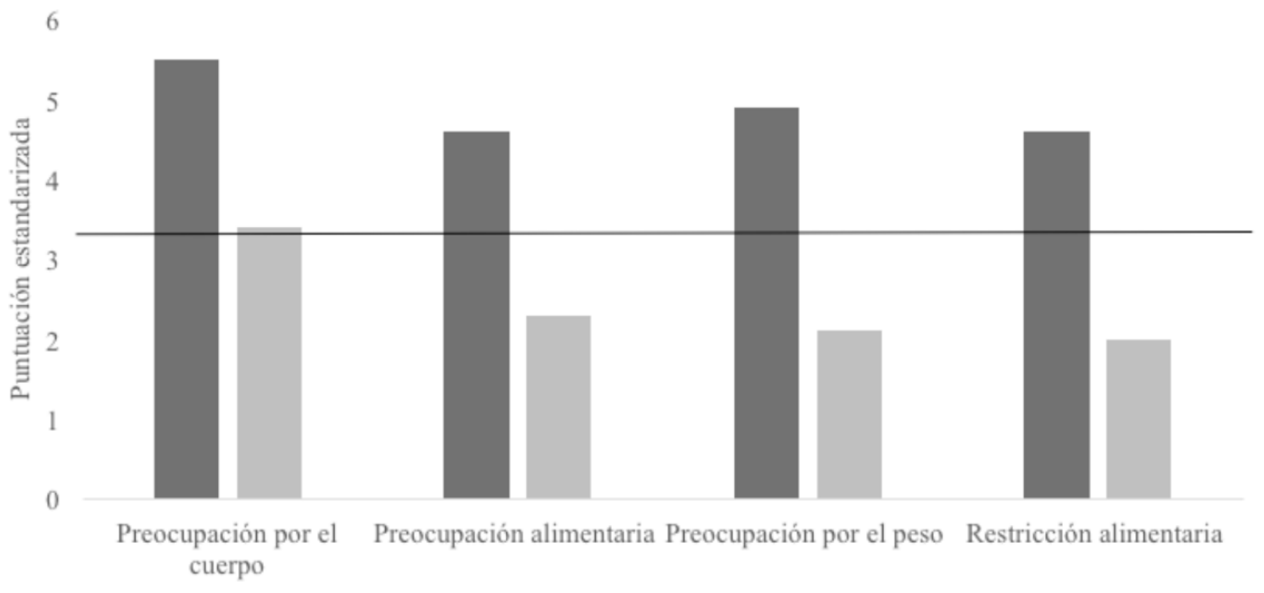

Subescalas

-PRE $M$ POST

\section{Análisis cuantitativo}

Psicopatología alimentaria (EDE-Q). Durante la evaluación inicial, la paciente puntuó por encima del punto de corte (establecido en 4) en todas las subescalas: preocupación por el cuerpo, preocupación alimentaria, preocupación por el peso y restricción alimentaria. En los resultados POST se observa una disminución de la puntuación en todas ella, situándose por debajo del punto de corte (4).

Además, el ítem del EDE-Q "frecuencia de atracones" disminuye desde una puntuación de 6 , equivalente a todos los días, hasta desaparecer por completo, con una puntuación igual a 0 en la fase post. En cuanto a la "frecuencia de purgas" disminuyen de una puntuación de 6 (todos los días) a una frecuencia esporádica (1-5 días).

Sintomatología bulímica (BITE). En la fase PRE, la paciente obtuvo una puntuación directa (29 puntos) equivalente a presencia de un patrón alimentario alterado y presencia de episodios de sobreingestas; la puntuación en severidad fue alta, 17 puntos; la puntuación global fue 46, lo cual indica diagnóstico de Bulimia Nerviosa. En la fase POST, las puntuaciones descienden por debajo de los puntos de corte para el diagnóstico de BN. 
Figura 3. Puntuaciones PRE-POST obtenidas en Sintomatología Bulímica, medidas a través del cuestionario BITE.

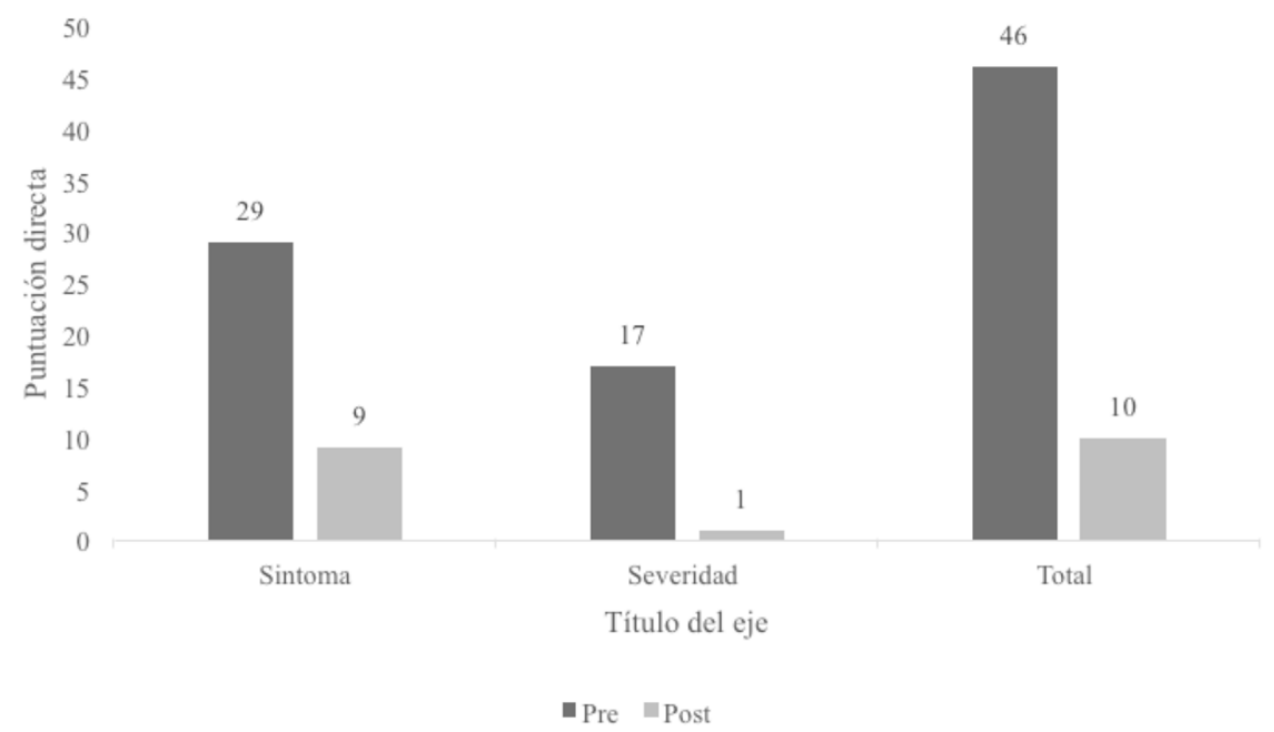

Asimismo, se midió la evolución de una serie de las variables psicológicas relacionadas con la psicopatología principal:

Sintomatología depresiva (a través del BDI-II). En la evaluación inicial, la paciente mostró una puntuación directa de 34, equivalente a depresión grave; mientras que en la evaluación POST se observa un descenso de 18 puntos, situándose en depresión leve.

Regulación emocional (a través del DERS). La paciente muestra dificultades de regulación emocional (puntuación directa total de 88) en comparación con la media obtenida por mujeres de la población general (PD: 59,1). Tras la intervención, se observa una disminución de dichas dificultades, obteniendo en la fase POST una puntuación directa de 62 .

Sintomatología ansiosa (a través de STAI). La paciente presentaba ansiedad elevada en el momento de la evaluación inicial (A/E), situándose la ansiedad estado en el percentil 75, y alta propensión ansiosa de manera estable (A/R), situándose la puntuación en ansiedad rasgo en el percentil 85. En la evaluación post-tratamiento, se observa que los niveles descienden en ambos casos, hasta niveles medios comparados con la población general, percentil 50 en ambos casos.

\section{Discusión}

Para el tratamiento de este caso se ha llevado a cabo una intervención cognitivo- conductual, siguiendo una serie de pasos: evaluación, formulación del caso (análisis funcional (AF)), elección de técnicas de intervención en función del AF y la planificación del tratamiento y aplicación del mismo. El caso de L.P. encaja con los criterios diagnósticos de la Bulimia 
Nerviosa, según criterios DSM-5 (APA, 2013). A continuación, se valoran los resultados del tratamiento.

La efectividad hace referencia al grado en que un tratamiento logra los objetivos terapéuticos en la práctica clínica habitual (Ballesteros, 2015), es decir, apunta hacia el éxito social y la satisfacción del paciente (Ferro y Vives, 2004). En este sentido, se puede concluir que el tratamiento ha sido efectivo, puesto que se observaron mejorías notables en los objetivos terapéuticos propuestos: se estableció una correcta adherencia al tratamiento, que se observa en la implicación de la paciente en el mismo (asistencia y realización de tareas), la paciente estableció un patrón de cinco ingestas diarias, se redujo las conductas compensatorias, se disminuyó la cantidad y frecuencia de los episodios de sobreingestas/atracones, y por último, la paciente experimenta una mejoría en su autoconcepto o definición de su identidad basado otros valores. La eficiencia hace referencia a la relación coste-beneficio de la propia intervención (Ballesteros, 2015), en este sentido, el tratamiento no ha supuesto ningún coste económico para la paciente, ni tampoco para su familia o la comunidad, ya que ha sido asumido completamente por el equipo terapéutico. Por otro lado, la paciente se ha beneficiado del tratamiento al experimentar mejoría tanto en las áreas objeto de tratamiento como en otras áreas de modo indirecto (estado de ánimo, ansiedad, manejo emocional, entre otras), y de igual modo ha notado mejoría en sus relaciones sociales. Por todo ello, se puede concluir que la intervención ha sido eficiente. Por último, la eficacia hace referencia a los logros de los objetivos terapéuticos en condiciones propias de la investigación (Ballesteros, 2015). Por tanto, ésta no puede ser medida mediante diseño de caso único al no realizarse comparación alguna. No obstante, existe un extenso cuerpo de investigación que avala que, hasta la fecha, el tratamiento más eficaz para la $\mathrm{BN}$ es la terapia cognitivo-conductual (Agras, Walsh, Fairburn et al., 2000).

En cuanto a la generalización de las conductas aprendidas, podemos considerar que un factor fundamental ha sido la puesta en práctica de las técnicas aprendidas en su entorno cotidiano. Por ejemplo, L.P generaliza la técnica de respiración diafragmática a situaciones estresantes que no estaban directamente relacionadas con la comida. También se observó la generalización de la reestructuración de pensamientos en situaciones distintas a las de discusión (ej: situaciones sociales).

Este trabajo cuenta con una serie de limitaciones que han de ser mencionadas. La principal limitación de la intervención ha sido la duración de esta, estipulada en cinco meses. Este periodo, en principio, es suficiente para tratar la sintomatología central del trastorno (Fairburn, 2008), sin embargo, al dedicar las primeras sesiones a tareas de motivación al cambio, junto a la no asistencia de la paciente a un par de sesiones, ha provocado que algunas partes de la sintomatología central no hayan sido abordadas. La justificación de esta elección es que, sin la correcta adherencia al tratamiento, el abordaje de la sintomatología central de la BN no sería posible. En cuanto al resto de conductas problema relacionadas con la sintomatología alimentaria, se valoró la interferencia de éstas en la vida de la paciente y se estableció el orden de abordaje. Por último, existen otras áreas en las que la paciente tiene dificultades, como es la relación familiar. Esta última área es de gran relevancia, pues la paciente convive en el seno de una familia disfuncional. Parece imprescindible una evaluación en profundidad de esta área de cara al mantenimiento a lo largo del tiempo de los resultados obtenidos. 


\section{Agradecimientos}

Al Centro Item, especializado en Trastornos del Comportamiento Alimentario, por mi formación clínica en el Prácticum extensivo del MPGS. Agradezco el aprendizaje clínico realizado en el curso de esta intervención.

\section{Referencias}

Agras, W.S., Walsh, B.T., Fairburn, C.G., Wilson, G.T y Kraemer, H.C. (2000). A Multicenter Comparison of Cognitive-Behavioral Therapy and Interpersonal Psychotherapy for Bulimia Nervosa. Archives of General Psychiatry, 57 (5), 459- 466.

American Psychiatric Association (APA). (2000). Practice guideline for the treatment of eating disorders. Washington, DC: APA.

American Psychiatric Association (APA). (2013). Diagnostic and Statistical Manual of Mental Disorders-5. Washington, DC: APA.

Ballesteros, F. (2015). Efectividad de los tratamientos psicológicos en el ámbito aplicado. Barcelona: Planeta.

Beck, A. T., Steer, R. A., Brown, G. K. (1996). Manual for the Beck Depression Inventory-II. San Antonio, TX: Psychological Corporation.

Buela-Casal G, Guillén-Riquelme A y Seisdedos-Cubero N. (2011). Cuestionario de ansiedad estado-rasgo. Octava edición. Madrid: TEA.

Calvo, R. (2011). Comprendiendo y superando la cronicidad en Trastornos de la Conducta Alimentaria. Acción Psicológica, $8(1), 35-56$.

Fairburn, C. G (1995). La superación de los atracones de comida. Barcelona: Paidós Ibérica.

Fairburn, C. G (2008). Cognitive-behaviour therapy and eating disorders. New York: Guilford Press.

Fairburn, C. G., Cooper, Z., Doll, H. A., O'Connor, M. E., Palmer, R. L., y Dalle, R. (2013). Enhanced cognitive behaviour therapy for adults with anorexia nervosa: A UK-Italy study. Behaviour Research and Therapy, 51 (1), 2-8.

Fairburn, C. G., y Beglin, S. J. (1994). Assessment of eating disorders: Interview or self-report questionnaire? International Journal of Eating Disorder, 16 (4), 363-370.

Fairburn, C.G., Cooper, Z., y Shafran, R. (2003). Cognitive-Behaviour therapy for eating disorders: a "transdiagnostic" theory and treatment. Behaviour Research and Therapy, 41 (1), 509-521.

Ferro, R., y Vives, C. (2004). Un análisis de los conceptos de efectividad, eficacia y eficiencia en psicología. Panace@, 5 (16), 97-99.

Gandarillas, A., Zorrilla, B., Sepúlveda, A. R., Muñoz, E. (2003) Trastornos del Comportamiento Alimentario: prevalencia de casos clínicos en mujeres adolescentes de la Comunidad de Madrid. Madrid: Documentos Técnico de Salud Pública, $n^{\circ} 85$. Instituto de Salud Pública.

Graell, M., Villaseñor, A., y Morandé, G. (2014). Clínica y evaluación de los trastornos de alimentación de la edad prepuberal. En Morandé, G., Graell, M., Blanco, M.A. (Coords.) Trastornos de la conducta alimentaria y obesidad. Un enfoque integral. (pp. 123-133). Madrid: Panamericana.

Gratz, K.L. y Roemer, L. (2004). Multidimensional Assessment of Emotion Regulation and Dysregulation: Development, Factor Structure, and Initial Validation of the Difficulties in Emotion Regulation Scale. Journal of Psychopathology and Behavioral Assessment, 26 (1), 41-54.

Henderson, M y Freeman, P.L. (1987). A Self-rating Scale for Bulimia. The BITE. British Journal Psychiatry, 150, 18-24.

Herpertz-Dahlmann B. (2009). Adolescent eating disorders: definitions, symptomatology, epidemiology and comorbidity. Child and Adolescent Psychiatric Clinics of North America, 18 (1), 31-47. 
Hervás, G. y Jódar, R. (2008). Adaptación al castellano de la Escala de Dificultades en la Regulación Emocional. Clínica y Salud, 19 (2), 139-156.

Hoek, H., y Van Hoeken, D. (2003). Review of the prevalence and incidence of Eating Disorders. International Journal of Eating Disorders, 25 (34), 383-396.

Instituto Nacional de la Salud (1995). Protocolo de los trastornos del comportamiento alimentario. Madrid: INSALUD. Ministerio de Sanidad y Consumo.

Instituto Nacional de Salud (2000). Trastornos del comportamiento alimentario. Criterios de ordenación de recursos y actividades. Madrid: INSALUD. Ministerio de Sanidad y Consumo.

Keel, P.K. y Brown, T.A. (2010). Update on course and outcome in eating disorders. International Journal of Eating Disorders, 43 (3), 195-204.

Miller, W. R. y Rollnick, S. (2015). La entrevista motivacional. Ayudar a las personas a cambiar. (3²Ed.) Barcelona: Paidós Ibérica.

Miller, W.R., y Rollnick, S. (1991). Motivational interviewing: Preparing people to change addictive behavior. New York: Guilford Press.

Mitchell, J. E., Agras, S., Crow, S., Halmi, K., Fairburn, C. G., Bryson, S., y Kraemer, H. (2011). Stepped care and cognitive-behavioural therapy for bulimia nervosa: randomised trial. The British Journal of Psychiatry, 198 (5), 391-397.

Morandé, G. (2014). Introducción. En Morandé, G., Graell, M., Blanco, M.A. (Coords.) Trastornos de la conducta alimentaria y obesidad. Un enfoque integral. (pp.5-10) Madrid: Panamericana.

National Institute for Health and Care Excellence (NICE). (2017). Eating Disorders: recognition and treatment. Nice Guideline (NG69). Recuperado de: https://www.nice.org.uk/guidance/ng69

Peláez, M. A., Labrador, F. J. y Raich, R. M. (2005). Prevalencia de los trastornos de la conducta alimentaria: consideraciones metodológicas. International Journal of Psychology and Psychological Therapy, 5 (2), 135-148.

Peláez, M. A., Labrador, F. J., y Raich, R. (2013). Norms for the Spanish version of the Eating Disorders Examination Questionnaire (S-EDE-0). Psicothema, 25 (1), 107-114.

Peláez, M. A., Raich, R. M., y Labrador, F. J. (2010). Trastornos de la conducta alimentaria en España: Revisión de estudios epidemiológicos. Revista mexicana de trastornos alimentarios, 1 (1), 62-75.

Prochaska, J. 0., y DiClemente, C. C. (1984). The transtheoretical approach: Crossing traditional boundaries of change. Homewood, IL: Dorsey Press.

Rivas, T., Bersabé, R. y Jiménez, M. (2004). Fiabilidad y validez del Test de Investigación Bulímica de Edimburgo (BITE) en una muestra de adolescentes españoles. Psicología Conductual, 12, 447-461.

Sanz, J., Navarro, M.E. y Vázquez, C. (2003). Adaptación española del Inventario para la Depresión de Beck-II (BDI-II): 1. Propiedades psicométricas en estudiantes universitarios. Análisis y modificación de conducta, 29 (124), 239-288.

Serpell, L., Treasure, J., Teasdale, J. y Sullivan, V. (1999). Anorexia nervosa: Friend or foe? International Journal of Eating Disorders, 25(2), 177-186.

Smink, FRE., Van Hoeken, D y Hoek, HW. (2012) Epidemiology of eating disorders: incidence, prevalence and mortality rates. Current Psychiatry Report, 14 (4), 406-414.

Spielberger, C. D., Gorsuch, R. y Lushene, R. (1970). Manual for the State-Trait Anxiety Inventory. Palo Alto: Consulting Psychologist Press.

Treasure, J. L. y Schmidt, U. H. (1997). A Clinician's Guide to management of bulimia nervosa (Motivational Enhancement Therapy for Bulimia Nervosa). Hove: Psychology Press.

Treasure, J., Claudino, A. M. y Zucker, N. (2010). Eating disorders. The Lancet, 375 (9714), 583-93.

Turón, V. (2011). Evolución de los TCA. ¿Es posible la curación? En R. Baztarrica, L. Beato, A. Blanco, R. Calvo, J. Casas, G. Faus, M. Faya y M. Graell. (Ed). Controversias sobre los trastornos alimentarios. (pp. 119-133). Madrid: IMC. Recuperado de http://www.institutotomaspascual.es/publicacionesactividad/publi/Libro_Controversias_Trastornos_Alimentarios.pdf 